\title{
A New Class of $\psi$-Caputo Fractional Differential Equations and Inclusion
}

\author{
Wafa Shammakh (D, Hadeel Z. Alzumi ${ }^{D}$, and Bushra A. AlQahtani \\ University of Jeddah, College of Science, Department of Mathematics, Jeddah, Saudi Arabia \\ Correspondence should be addressed to Hadeel Z. Alzumi; hzalzumi@uj.edu.sa
}

Received 21 November 2020; Accepted 21 December 2020; Published 18 January 2021

Academic Editor: Ching-Feng Wen

Copyright (c) 2021 Wafa Shammakh et al. This is an open access article distributed under the Creative Commons Attribution License, which permits unrestricted use, distribution, and reproduction in any medium, provided the original work is properly cited.

In the present research work, we investigate the existence of a solution for new boundary value problems involving fractional differential equations with $\psi$-Caputo fractional derivative supplemented with nonlocal multipoint, Riemann-Stieltjes integral and $\psi$-Riemann-Liouville fractional integral operator of order $\gamma$ boundary conditions. Also, we study the existence result for the inclusion case. Our results are based on the modern tools of the fixed-point theory. To illustrate our results, we provide examples.

\section{Introduction}

Fractional calculus has played a very important role in different areas of research (see $[1,2]$ and the references cited therein). Consequently, fractional differential equations have grasped the interest of many researchers working in diverse applications [3-6]. Recently, several researchers have tried to propose different types of fractional operators that deal with derivatives and integrals of arbitrary orders and their applications. For instance, Kilbas et al. in [2] introduced the properties of fractional integrals and fractional derivatives concerning another function. Some generalized fractional integral and differential operators and their properties were introduced by Agrawal in [7]. Very recently, Almeida in [8] presented a new type of fractional differentiation operator, the so-called $\psi$-Caputo fractional operator, and extended work of Caputo [2,9]. Almeida et al. in $[10,11]$ investigated the existence and uniqueness of the results of nonlinear fractional differential equations involving a Caputo-type fractional derivative with respect to another function, employing the fixed-point theorem and Picard iteration method. Numerous interesting results concerning the existence, uniqueness, and stability of initial value problems and boundary value problems for fractional differential equations with $\psi$-Caputo fractional derivatives by applying different types of fixed-point techniques were obtained by Abdo et al. [12, 13], Vivek et al. [14], and Wahash et al. [15]. An important application that is controlled by the theory of $\psi$-fractional differentiation can be found in [16].

In this paper, we investigate a new boundary value problem of fractional differential equations supplemented with nonlocal multipoint, Riemann-Stieltjes integral fractional boundary conditions involving Riemann-Liouville fractional integral operator of order $\gamma>0$ with respect to function $\psi$ given by the form

$$
\left\{\begin{array}{l}
{ }^{c} D_{0^{+}, \psi}^{\alpha}\left({ }^{c} D_{0^{+}, \psi}^{\beta} u(t)+h(t, u(t))\right)=f(t, u(t)), \quad 0<\alpha, \beta \leq 1, \\
u(0)=a_{1} \Omega[u], \\
u(1)=a_{2} \theta[u]+a_{3} \sum_{i=1}^{m} \mu_{i} I_{0^{+}, \psi}^{\gamma} u\left(\eta_{i}\right),
\end{array}\right.
$$

where ${ }^{c} D_{0^{+}, \psi}^{\alpha}$ and ${ }^{c} D_{0^{+}, \psi}^{\beta}$ denotes the $\psi$-Caputo fractional derivatives of orders $0<\alpha \leqslant 1$ and $0<\beta \leqslant 1$, respectively, $I_{0^{+}, \psi}^{\gamma}$ is the $\psi$-Riemann-Liouville fractional integral operator of order $\gamma>0$. The functions $h:[0,1] \times \mathbb{R} \longrightarrow \times \mathbb{R}$ and $f:[0,1] \times$ $\mathbb{R} \longrightarrow \times \mathbb{R}$ are continuous, whereas $\Omega[u]=\int_{0}^{1} u(s) \mathrm{d} A_{1}(s)$ and $\theta[u]=\int_{0}^{1} u(s) \mathrm{d} A_{2}(s)$ are Riemann-Stieltjes integral 
and $A_{i}(\cdot),(i=1,2)$ is a function of bounded variation. $a_{i}(i=$ $1,2,3)$ is a real constant, and $\mu_{i}, \eta_{i}(i=1, \ldots, m)$ are positive constants.

We also study the corresponding inclusion problem that is given by

$$
\left\{\begin{array}{l}
{ }^{c} D_{0^{+}, \psi}^{\alpha}\left({ }^{c} D_{0^{+}, \psi}^{\beta} u(t)+h(t, u(t)) \in F(t, u(t)), \quad 0<\alpha, \beta \leq 1,\right. \\
u(0)=a_{1} \Omega[u], \\
u(1)=a_{2} \theta[u]+a_{3} \sum_{i=1}^{m} \mu_{i} I_{0^{+}, \psi}^{\gamma} u\left(\eta_{i}\right),
\end{array}\right.
$$

where $F: C([0,1], \mathbb{R}) \longrightarrow \mathscr{P}(\mathbb{R})$ is a multivalued function, where $\mathscr{P}(\mathbb{R})$ is the family of all subsets of $\mathbb{R}$ and the other quantities are the same as defined in problem (1).

Notice that this Riemann-Stieltjes integral fractional boundary conditions arise in manifold applications of computational fluid dynamics, distribution methods, and so forth (for example, see $[17,18]$ ).
This paper is organized as follows. In Section 2, we recall some preliminary results and some related definitions. In Section 3, we discuss the existence results of solutions by relying on Krasnoselskii fixed-point theorem and Leray-Schauder nonlinear alternative. Also, we present an example. Finally, we describe the inclusion case and deduce the existence of solutions by applying Krasnoselskii's multivalued fixed-point theorem in Section 4.

\section{Preliminaries}

For the convenience of the reader, we present here some necessary basic definitions, lemmas, and results which are used throughout this paper $[2,8,10,12,19,20]$.

Definition 1. Let $[a, b]$ be a finite interval and $0 \leq \varepsilon<1$ and $\mathrm{AC}[a, b]$ be the set of absolute continuous functions on $[a, b]$. Then, we define

$$
\begin{aligned}
& \operatorname{AC}_{\gamma}^{n}[a, b]=\left\{f:[a, b] \longrightarrow \mathrm{C} \text { and } \gamma^{n-1} f \in \mathrm{AC}[a, b], \gamma=x^{1-\rho} \frac{\mathrm{d}}{\mathrm{d} x}\right\}, \\
& \operatorname{AC}_{\gamma}^{1}[a, b]=\mathrm{AC}[a, b], \\
& C_{\gamma, \varepsilon}^{n}[a, b]=\left\{f:[a, b] \longrightarrow \mathrm{C} \text { and } \gamma^{n-1} f \in C[a, b], \gamma^{n} f \in C_{\varepsilon, \rho}, \gamma=x^{1-\rho} \frac{\mathrm{d}}{\mathrm{d} x}\right\},
\end{aligned}
$$

endowed with the norm $\|f\|_{C^{n}}=\sum_{k=0}^{n-1}\left\|\gamma^{k} f\right\|_{C}+\left\|\gamma^{n} f\right\|_{C}$. The convention $C_{\gamma, 0}^{n}[a, b]=C_{\gamma}^{n}[a, b]$ endowed with the norm $\|f\|_{C_{\gamma}^{n}}=\sum_{k=0}^{n}\left\|\gamma^{k} f\right\|_{C}$ is used.

Definition 2. Let $\alpha>0, h$ be an integrable function defined on $[a, b]$, and $\psi \in C^{n}[a, b]$ be an increasing differentiable function such that $\psi(t) \neq 0$ for all $t \in[a, b]$. The left-sided $\psi$-Riemann-Liouville fractional integral of order $\alpha$ of a function $h$ is given by

$$
I_{0^{+}, \psi}^{\alpha} h(t)=\frac{1}{\Gamma(\alpha)} \int_{0}^{t}(\psi(t)-\psi(s))^{\alpha-1} \psi(s) h(s) \mathrm{d} s .
$$

Definition 3. Let $n-1<\alpha<n, h:[a, b] \longrightarrow \mathbb{R}$ be an integrable function and $\psi \in C_{,}^{n}[a, b]$ be an increasing differentiable function such that $\psi(t) \neq 0$ for all $t \in[a, b]$. The leftsided $\psi$-Riemann-Liouville fractional derivative of order $\alpha$ of a function $h$ is defined by

$$
D_{0^{+}, \psi}^{\alpha} h(t)=\left[\frac{1}{\psi(t)} \frac{\mathrm{d}}{\mathrm{d} t}\right]^{n} I_{0^{+}, \psi}^{n-\alpha} h(t)
$$

where $n=[\alpha]+1$ and $[\alpha]$ denote the integer part of the real number $\alpha$.

Definition 4. Let $n-1<\alpha<n, h \in C^{n-1}[a, b], \quad$ and $\psi \in C^{n},[a, b]$ be an increasing differentiable function such that $\psi(t) \neq 0$ for all $t \in[a, b]$. The left-sided $\psi$-Caputo fractional derivative of order $\alpha$ of a function $h$ is defined by

$$
{ }^{c} D_{0^{+}, \psi}^{\alpha} h(t)=D_{0^{+}, \psi}^{\alpha}\left[h(t)-\sum_{k=0}^{n-1} \frac{h_{\psi}^{[k]}(0)}{k !}(\psi(t)-\psi(0))^{k}\right],
$$

where $h_{\psi}^{[k]}(t)=[(1 / \psi(t))(\mathrm{d} / \mathrm{d} t)]^{k} h(t)$ and $n=[\alpha]+1$ for $\alpha \notin \mathbb{N}$ and $n=\alpha$ for $\alpha \in \mathbb{N}$. Furthermore, if $h \in C^{n}[a, b]$ and $\alpha \notin \mathbb{N}$, then

$$
{ }^{c} D_{0^{+}, \psi}^{\alpha} h(t)=I_{0^{+}, \psi}^{n-\alpha}\left[\frac{1}{\psi(t)} \frac{\mathrm{d}}{\mathrm{d} t}\right]^{n} h(t)=\frac{1}{\Gamma(n-\alpha)} \int_{0}^{t}(\psi(t)-\psi(s))^{n-\alpha-1} \psi(s) h_{\psi}^{[n]}(s) \mathrm{d} s .
$$


Thus, if $n=[\alpha] \in \mathbb{N}$, one has

$$
{ }^{c} D_{0^{+}, \psi}^{\alpha} h(t)=h_{\psi}^{[n]}(t) .
$$

Lemma 1. Given a function $h \in A C_{\psi}^{n}([0,1])$ and $\alpha \in \mathbb{R}^{+}$, then

$$
I_{0^{+}, \psi}^{\alpha}{ }^{c} D_{0^{+}, \psi}^{\alpha} h(t)=h(t)-\sum_{k=0}^{n-1} \frac{\left(\delta_{\psi}^{k} h\right)(0)}{k !}(\psi(t)-\psi(0))^{k} .
$$

Lemma 2. Let $\alpha, \beta>0$ and $h:[a, b] \longrightarrow \mathbb{R}$; then,

$$
\begin{aligned}
& \text { (i) } I_{0^{+}, \psi}^{\alpha}[\psi(t)-\psi(0)]^{\beta-1}=((\Gamma(\beta)) /(\Gamma(\alpha+\beta))) \\
& {[\psi(t)-\psi(0)]^{\alpha+\beta-1}} \\
& \text { (ii) }{ }^{c} D_{0^{+}, \psi}^{\alpha}[\psi(t)-\psi(0)]^{\beta-1}=((\Gamma(\beta)) /(\Gamma(\beta-\alpha))) \\
& {[\psi(t)-\psi(0)]^{\beta-\alpha-1}} \\
& \text { (iii) }{ }^{c} D_{0^{+}, \psi}^{\alpha}[\psi(t)-\psi(0)]^{k}=0, \forall k \in\{0,1, \ldots, n-1\} \text {, } \\
& n \in \mathbb{N} \\
& \text { (iv) } I_{0^{+}, \psi}^{\alpha} I_{0^{+}, \psi}^{\beta} h(t)=I_{0^{+}, \psi}^{\alpha+\beta} h(t)
\end{aligned}
$$

The existence of solutions of problem (1) relies on the following fixed-point theorems [21, 22].

Theorem 1 (Krasnoselskii's fixed-point theorem). Let $\mathfrak{p}$ be a closed, convex, bounded, and nonempty subset of a Banach space $X$. Let $T_{1}$ and $T_{2}$ be operators such that

(i) $T_{1}\left(u_{1}\right)+T_{2}\left(u_{2}\right)$ belong to $\mathfrak{p}$ whenever $u_{1}, u_{2} \in \mathfrak{p}$

(ii) $T_{1}$ is compact and $T_{2}$ is a contraction mapping

Then, there exist $u_{0} \in \mathfrak{p}$ such that $u_{0}=T_{1}\left(u_{0}\right)+T_{2}\left(u_{0}\right)$.
Theorem 2 (Leray-Schauder fixed-point theorem). Let $C$ be a closed and convex subset of a Banach space $E$ and $U$ be an open subset of $C$ with $0 \in U$. Suppose that $\mathscr{V}: \bar{U} \longrightarrow C$ is a continuous, compact (that is, $\mathscr{V}(\bar{U})$ is a relatively compact subset of C) map. Then, either

(i) $\mathscr{V}$ has a fixed point in $\bar{U}$ or

(ii) there are $u \in \partial U$ (the boundary of $U$ in $C$ ) and $\lambda \in(0,1)$ with $u=\lambda \mathscr{V}(u)$

For computational convenience, we set the following:

$$
\begin{aligned}
& \varphi=1-a_{3} \sum_{i=1}^{m} \mu_{i} \frac{\left(\psi\left(\eta_{i}\right)-\psi(0)\right)^{\gamma}}{\Gamma(\gamma+1)}, \\
& \zeta=a_{3} \sum_{i=1}^{m} \mu_{i} \frac{\left(\psi\left(\eta_{i}\right)-\psi(0)\right)^{\beta+\gamma}}{\Gamma(\beta+\gamma+1)}-\frac{(\psi(1)-\psi(0))^{\beta}}{\Gamma(\beta+1)} .
\end{aligned}
$$

Lemma 3. Let $y, g \in C([0,1], \mathbb{R})$; then, the linear $\psi$-fractional differential equation

$$
\left\{\begin{array}{l}
{ }^{c} D_{0^{+}, \psi}^{\alpha}\left({ }^{c} D_{0^{+}, \psi}^{\alpha} u(t)+g(t)\right)=y(t), \\
u(0)=a_{1} \Omega[u], \\
u(1)=a_{2} \theta[u]+a_{3} \sum_{i=1}^{m} \mu_{i} I_{0^{+}, \psi}^{\gamma} u\left(\eta_{i}\right),
\end{array}\right.
$$

has a solution $u(t)$ on $[0,1]$ given by

$$
\begin{aligned}
u(t)= & I_{0^{+}, \psi}^{\alpha+\beta} y(t)-I_{0^{+}, \psi}^{\beta} g(t)+\frac{(\psi(t)-\psi(0))^{\beta}}{\zeta \Gamma(\beta+1)}\left[I_{0^{+}, \psi}^{\alpha+\beta} y(1)-I_{0^{+}, \psi}^{\beta} g(1)\right. \\
& \left.+a_{3} \sum_{i=1}^{m} \mu_{i}\left(I_{0^{+}, \psi}^{\alpha+\beta+\gamma} y\left(\eta_{i}\right)-I_{0^{+}, \psi}^{\beta+\gamma} g\left(\eta_{i}\right)\right)+a_{1} \varphi \Omega[u]-a_{2} \theta[u]\right]+a_{1} \Omega[u] .
\end{aligned}
$$

Proof. We apply $\psi$-Riemann-Liouville fractional integral of order $\alpha$ to both sides of the linear $\psi$-fractional differential equation:

$$
{ }^{c} D_{0^{+}, \psi}^{\alpha}\left({ }^{c} D_{0^{+}, \psi}^{\beta} u(t)+g(t)\right)=y(t), \quad 0<\alpha, \beta \leq 1 .
$$

We obtain

$$
{ }^{c} D_{0^{+}, \psi}^{\beta} u(t)+g(t)=I_{0^{+}, \psi}^{\alpha} y(t)+c_{1} .
$$

Next, applying $\psi$-Riemann-Liouville fractional integral of order $\beta$ to both sides of (14), we obtain

$$
u(t)=-I_{0^{+}, \psi}^{\beta} g(t)+I_{0^{+}, \psi}^{\alpha+\beta} y(t)+I_{0^{+}, \psi}^{\beta} c_{1}+c_{2},
$$

where $c_{1}$ and $c_{2}$ are arbitrary constants. By Definition 1, general solution (15) can be written as

$$
u(t)=I_{0^{+}, \psi}^{\alpha+\beta} y(t)-I_{0^{+}, \psi}^{\beta} g(t)+\frac{c_{1}}{\Gamma(\beta+1)}(\psi(t)-\psi(0))^{\beta}+c_{2} .
$$

Using the boundary condition $u(0)=a_{1} \Omega[u]$, we obtain $c_{2}=a_{1} \Omega[u]$. Thus, (16) takes the form

$$
u(t)=I_{0^{+}, \psi}^{\alpha+\beta} y(t)-I_{0^{+}, \psi}^{\beta} g(t)+\frac{c_{1}}{\Gamma(\beta+1)}(\psi(t)-\psi(0))^{\beta}+a_{1} \Omega[u] .
$$
obtain

Applying the operator $I_{0^{+}, \psi}^{\gamma}, \gamma>0$, on equation (17), we

$$
\begin{aligned}
I_{0^{+}, \psi}^{\gamma} u(t)= & I_{0^{+}, \psi}^{\alpha+\beta+\gamma} y(t)-I_{0^{+}, \psi}^{\beta+\gamma} g(t)+\frac{c_{1}(\psi(t)-\psi(0))^{\beta+\gamma}}{\Gamma(\beta+\gamma+1)} \\
& +\frac{a_{1} \Omega[u](\psi(t)-\psi(0))^{\gamma}}{\Gamma(\gamma+1)} .
\end{aligned}
$$


Using the boundary condition $u(1)=a_{2} \theta[u]+$ $a_{3} \sum_{i=1}^{m} \mu_{i} I_{0^{+}, \psi}^{\gamma} u\left(\eta_{i}\right)$, we find that

$$
c_{1}=\frac{1}{\zeta}\left[I_{0^{+}, \psi}^{\alpha+\beta} y(1)-I_{0^{+}, \psi}^{\beta} g(1)-a_{2} \theta[u]+a_{1} \Omega[u]\left(1-a_{3} \sum_{i=1}^{m} \mu_{i} \frac{\left(\psi\left(\eta_{i}\right)-\psi(0)\right)^{\gamma}}{\Gamma(\gamma+1)}\right)+a_{3} \sum_{i=1}^{m} \mu_{i}\left(I_{0^{+}, \psi}^{\alpha+\beta+\gamma} y\left(\eta_{i}\right)-I_{0^{+}, \psi}^{\beta+\gamma} g\left(\eta_{i}\right)\right)\right] .
$$

Inserting the value of $c_{2}$ in (17) yields solution (12). The converse follows by direct computation.

\section{Main Results}

In this section, we prove the existence of solutions of problem (1). We shall assume that $f$ and $h$ are in the Banach space $C([0,1], \mathbb{R})$. Let $U=\{u: u \in C([0,1], \mathbb{R})\}$ denote the Banach space of all continuous functions on $[0,1]$ into $\mathbb{R}$ endowed with the norm $\|u\|=\sup \{|u(t)|: t \in[0,1]\}$. Here, we define an operator $\mathscr{T}: U \longrightarrow U$ associated with problem (1) by

$$
\begin{aligned}
(\mathscr{T} u)(t)= & I_{0^{+}, \psi}^{\alpha+\beta} f(t, u(t))-I_{0^{+}, \psi}^{\beta} h(t, u(t))+\frac{(\psi(t)-\psi(0))^{\beta}}{\zeta \Gamma(\beta+1)}\left[I_{0^{+}, \psi}^{\alpha+\beta} f(1, u(1))\right. \\
& -I_{0^{+}, \psi}^{\beta} h(1, u(1))+a_{3} \sum_{i=1}^{m} \mu_{i}\left(I_{0^{+}, \psi}^{\alpha+\beta+\gamma} f\left(\eta_{i}, u\left(\eta_{i}\right)\right)-I_{0^{+}, \psi}^{\beta+\gamma} h\left(\eta_{i}, u\left(\eta_{i}\right)\right)\right) \\
& \left.+a_{1} \varphi \Omega[u]-a_{2} \theta[u]\right]+a_{1} \Omega[u] .
\end{aligned}
$$

Therefore, problem (1) has a solution if and only if the operator $\mathscr{T}$ has a fixed point.
For computational convenience, we introduce the notations

$$
\begin{aligned}
\mathscr{G}= & \frac{(\psi(1)-\psi(0))^{\alpha+\beta}}{\Gamma(\alpha+\beta+1)}-\frac{(\psi(1)-\psi(0))^{\beta}}{\Gamma(\beta+1)}+\frac{(\psi(1)-\psi(0))^{\beta}}{|\zeta| \Gamma(\alpha+\beta)} \\
& \cdot\left[\frac{(\psi(1)-\psi(0))^{\alpha+\beta}}{\Gamma(\alpha+\beta+1)}-\frac{(\psi(1)-\psi(0))^{\beta}}{\Gamma(\beta+1)}+\left|a_{3}\right| \sum_{i=1}^{m}\left|\mu_{i}\right|\left(\frac{\left(\psi\left(\eta_{i}\right)-\psi(0)\right)^{\alpha+\beta+\gamma}}{\Gamma(\alpha+\beta+\gamma+1)}\right.\right. \\
& \left.\left.-\frac{\left(\psi\left(\eta_{i}\right)-\psi(0)\right)^{\beta+\gamma}}{\Gamma(\beta+\gamma+1)}\right)\right], \\
\mathscr{G}_{1}= & a_{1} \Omega[u]\left(1+\frac{(\psi(1)-\psi(0))^{\beta}}{|\zeta| \Gamma(\alpha+\beta)}\right), \\
\mathscr{G}_{2}= & \left.\left.\frac{(\psi(1)-\psi(0))^{\beta}\left[\frac{(\psi(1)-\psi(0))^{\alpha+\beta}}{\Gamma(\alpha+\beta+1)}-\frac{(\psi(1)-\psi(0))^{\beta}}{\Gamma(\beta+1)}\right.}{|\zeta| \Gamma(\alpha+\beta)}-\frac{\left(\psi\left(\eta_{i}\right)-\psi(0)\right)^{\beta+\gamma}}{\Gamma(\beta+\gamma+1)}\right)\right] \\
& +\left|a_{3}\right| \sum_{i=1}^{m}\left|\mu_{i}\right|\left(\frac{\left(\psi\left(\eta_{i}\right)-\psi(0)\right)^{\alpha+\beta+\gamma}}{\Gamma(\alpha+\beta+\gamma+1)}\right. \\
\mathscr{G}_{3}= & a_{1}\left(1+\frac{(\psi(1)-\psi(0))^{\beta}}{|\zeta| \Gamma(\alpha+\beta)}\right) \int_{0}^{1} \mathrm{~d} A_{1}(s)-a_{2}\left(\frac{(\psi(1)-\psi(0))^{\beta}}{|\zeta| \Gamma(\alpha+\beta)}\right) \int_{0}^{1} \mathrm{~d} A_{2}(s)
\end{aligned}
$$


Now, we will state and prove the existence result via Krasnoselskii's fixed-point theorem.

Theorem 3. Let $f, h:[0,1] \times \mathbb{R} \longrightarrow \mathbb{R}$ be continuous functions satisfying the conditions:

(H1) $\quad|f(t, u)-f(t, \tilde{u})| \leq \rho_{1}\|u-\widetilde{u}\|$, and $\mid h(t, u)-$ $h(t, \widetilde{u}) \mid \leq \rho_{2}\|u-\widetilde{u}\|$ for all $t \in[0,1]$, every $u, \widetilde{u} \in \mathbb{R}$ and $\rho>0$, with $\rho \mathscr{G}_{2}+\mathscr{G}_{3}<1$ and $\rho=\max \left\{\rho_{1}, \rho_{2}\right\}$.

(H2) There exist continuous nonnegative functions $M_{1}, M_{2} \in C([0,1], \mathbb{R})$ such that $|f(t, u)| \leq M_{1}(t)$ and $|h(t, u)| \leq M_{2}(t), \forall(t, u) \in[0,1] \times \mathbb{R}$,

and $M=\max \left\{M_{1}, M_{2}\right\}$. Then, problem (1) has at least one solution on $[0,1]$.

Proof. For a positive number $\epsilon$, consider $B_{\epsilon}=\{u \in U$ : $\|u\| \leqslant \epsilon\}$, where $\epsilon \geq\|M\| \mathscr{G}+\mathscr{G}_{1}$, and we split $\mathscr{T}$ into two operators $\mathscr{T}_{1}$ and $\mathscr{T}_{2}$ where $\mathscr{T}=\mathscr{T}_{1}+\mathscr{T}_{2}$, on the bounded set $B_{\epsilon}$ by

$$
\begin{aligned}
\left(\mathscr{T}_{1} u\right)(t)= & I_{0^{+}, \psi}^{\alpha+\beta} f(t, u(t))-I_{0^{+}, \psi}^{\beta} h(t, u(t)), \\
\left(\mathscr{T}_{2} u\right)(t)= & \frac{(\psi(t)-\psi(0))^{\beta}}{\zeta \Gamma(\beta+1)}\left(I_{0^{+}, \psi}^{\alpha+\beta} f(1, u(1))-I_{0^{+}, \psi}^{\beta} h(1, u(1))+a_{3} \sum_{i=1}^{m} \mu_{i}\right. \\
& \left.\cdot\left(I_{0^{+}, \psi}^{\alpha+\beta+\gamma} f\left(\eta_{i}, u\left(\eta_{i}\right)\right)-I_{0^{+}, \psi}^{\beta+\gamma} h\left(\eta_{i}, u\left(\eta_{i}\right)\right)\right)+a_{1} \varphi \Omega[u]-a_{2} \theta[u]\right)+a_{1} \Omega[u] .
\end{aligned}
$$

For any $u \in B_{\epsilon}$, by using (H2), we have

$$
\begin{aligned}
& \left\|\mathscr{T}_{1} u+\mathscr{T}_{2} u\right\| \leq \sup _{t \in[0,1]}\left\{\int_{0}^{t} \frac{(\psi(t)-\psi(s))^{\alpha+\beta-1}}{\Gamma(\alpha+\beta)} \psi(s)|f(s, u(s))| \mathrm{d} s\right. \\
& -\int_{0}^{t} \frac{(\psi(t)-\psi(s))^{\beta-1}}{\Gamma(\beta)} \psi(s)|h(s, u(s))| \mathrm{d} s \\
& +\frac{(\psi(t)-\psi(0))^{\beta}}{|\zeta| \Gamma(\alpha+\beta)}\left[\int_{0}^{1} \frac{(\psi(1)-\psi(s))^{\alpha+\beta-1}}{\Gamma(\alpha+\beta)} \psi(s)|f(s, u(s))| \mathrm{d} s\right. \\
& -\int_{0}^{1} \frac{(\psi(1)-\psi(s))^{\beta-1}}{\Gamma(\beta)} \psi(s)|h(s, u(s))| \mathrm{d} s \\
& +\left|a_{3}\right| \sum_{i=1}^{m}\left|\mu_{i}\right|\left(\int_{0}^{\eta_{i}} \frac{\left(\psi\left(\eta_{i}\right)-\psi(s)\right)^{\alpha+\beta+\gamma-1}}{\Gamma(\alpha+\beta+\gamma)} \psi(s)|f(s, u(s))| \mathrm{d} s\right. \\
& \left.-\int_{0}^{\eta_{i}} \frac{\left(\psi\left(\eta_{i}\right)-\psi(s)\right)^{\beta+\gamma-1}}{\Gamma(\beta+\gamma)} \psi(s)|h(s, u(s))| s\right)+\left|a_{1} \varphi \Omega[u]\right| \\
& \left.\left.-\left|a_{2} \theta[u]\right|\right]+\left|a_{1} \Omega[u]\right|\right\} \\
& \leq\|M\| \sup _{t \in[0,1]}\left\{\frac{(\psi(t)-\psi(0))^{\alpha+\beta}}{\Gamma(\alpha+\beta+1)}-\frac{(\psi(t)-\psi(0))^{\beta}}{\Gamma(\beta+1)}+\frac{(\psi(t)-\psi(0))^{\beta}}{|\zeta| \Gamma(\alpha+\beta)}\right. \\
& \cdot\left[\frac{(\psi(1)-\psi(0))^{\alpha+\beta}}{\Gamma(\alpha+\beta+1)}-\frac{(\psi(1)-\psi(0))^{\beta}}{\Gamma(\beta+1)}+\left|a_{3}\right| \sum_{i=1}^{m}\left|\mu_{i}\right|\right. \\
& \left.\left.\left(\frac{\left(\psi\left(\eta_{i}\right)-\psi(0)\right)^{\alpha+\beta+\gamma}}{\Gamma(\alpha+\beta+\gamma+1)}-\frac{\left(\psi\left(\eta_{i}\right)-\psi(0)\right)^{\beta+\gamma}}{\Gamma(\beta+\gamma+1)}\right)\right]\right\} \\
& +a_{1} \Omega[u]\left(1+\frac{(\psi(1)-\psi(0))^{\beta}}{|\zeta| \Gamma(\alpha+\beta)}\right) \\
& \leq\|M\| \mathscr{G}+\mathscr{G}_{1} \leq \epsilon .
\end{aligned}
$$


Hence, $\mathscr{T}_{1} u+\mathscr{T}_{2} u \in B_{\epsilon}$. Next, we show that $\mathscr{T}_{2}$ is a contraction mapping. Let $u, \widetilde{u} \in \mathbb{R}$ and $t \in[0,1]$, so by using ( $\mathrm{H} 1)$, we have

$$
\begin{aligned}
& \left\|\mathscr{T}_{2} u-\mathscr{T}_{2} \tilde{u}\right\| \leq \sup _{t \in[0,1]}\left\{\frac{(\psi(t)-\psi(0))^{\beta}}{|\zeta| \Gamma(\alpha+\beta)}\right. \\
& {\left[\int_{0}^{1} \frac{(\psi(1)-\psi(s))^{\alpha+\beta-1}}{\Gamma(\alpha+\beta)} \psi(s)|f(s, u(s))-f(s, \widetilde{u}(s))| \mathrm{d} s\right.} \\
& -\int_{0}^{1} \frac{(\psi(1)-\psi(s))^{\beta-1}}{\Gamma(\beta)} \psi(s)|h(s, u(s))-h(s, \tilde{u}(s))| \mathrm{d} s \\
& +\left|a_{3}\right| \sum_{i=1}^{m}\left|\mu_{i}\right|\left(\int_{0}^{\eta_{i}} \frac{\left(\psi\left(\eta_{i}\right)-\psi(s)\right)^{\alpha+\beta+\gamma-1}}{\Gamma(\alpha+\beta+\gamma)} \psi(s)\right. \\
& \cdot|f(s, u(s))-f(s, \widetilde{u}(s))| \mathrm{d} s \\
& \left.-\int_{0}^{\eta_{i}} \frac{\left(\psi\left(\eta_{i}\right)-\psi(s)\right)^{\beta+\gamma-1}}{\Gamma(\beta+\gamma)} \psi(s)|h(s, u(s))-h(s, \tilde{u}(s))| \mathrm{d} s\right] \\
& \left.\left.+a_{1} \varphi(\Omega[u]-\Omega[\widetilde{u}])-a_{2}(\theta[u]-\theta[\widetilde{u}])\right)+a_{1}(\Omega[u]-\Omega[\widetilde{u}])\right\} \\
& \leq \rho\|u-\tilde{u}\| \sup _{t \in[0,1]}\left\{\frac { ( \psi ( t ) - \psi ( 0 ) ) ^ { \beta } } { | \zeta | \Gamma ( \alpha + \beta ) } \left[\frac{(\psi(1)-\psi(0))^{\alpha+\beta}}{\Gamma(\alpha+\beta+1)}\right.\right. \\
& -\frac{(\psi(1)-\psi(0))^{\beta}}{\Gamma(\beta+1)}+\left|a_{3}\right| \sum_{i=1}^{m}\left|\mu_{i}\right|\left(\frac{\left(\psi\left(\eta_{i}\right)-\psi(0)\right)^{\alpha+\beta+\gamma}}{\Gamma(\alpha+\beta+\gamma+1)}\right. \\
& \left.\left.\left.-\frac{\left(\psi\left(\eta_{i}\right)-\psi(0)\right)^{\beta+\gamma}}{\Gamma(\beta+\gamma+1)}\right)\right]\right\}+a_{1}\left(1+\frac{(\psi(1)-\psi(0))^{\beta}}{|\zeta| \Gamma(\alpha+\beta)}\right) \\
& \cdot \int_{0}^{1}|u-\tilde{u}| \mathrm{d} A_{1}(s)-a_{2}\left(\frac{(\psi(1)-\psi(0))^{\beta}}{|\zeta| \Gamma(\alpha+\beta)}\right) \\
& \cdot \int_{0}^{1}|u-\tilde{u}| \mathrm{d} A_{2}(s) \\
& \leq \rho\|u-\widetilde{u}\|\left\{\frac { ( \psi ( 1 ) - \psi ( 0 ) ) ^ { \beta } } { | \zeta | \Gamma ( \alpha + \beta ) } \left[\frac{(\psi(1)-\psi(0))^{\alpha+\beta}}{\Gamma(\alpha+\beta+1)}\right.\right. \\
& -\frac{(\psi(1)-\psi(0))^{\beta}}{\Gamma(\beta+1)}+\left|a_{3}\right| \sum_{i=1}^{m}\left|\mu_{i}\right|\left(\frac{\left(\psi\left(\eta_{i}\right)-\psi(0)\right)^{\alpha+\beta+\gamma}}{\Gamma(\alpha+\beta+\gamma+1)}\right. \\
& \left.\left.\left.-\frac{\left(\psi\left(\eta_{i}\right)-\psi(0)\right)^{\beta+\gamma}}{\Gamma(\beta+\gamma+1)}\right)\right]\right\}+a_{1}\left(1+\frac{(\psi(1)-\psi(0))^{\beta}}{|\zeta| \Gamma(\alpha+\beta)}\right) \\
& \cdot \int_{0}^{1}|u-\tilde{u}| d A_{1}(s)-a_{2}\left(\frac{(\psi(1)-\psi(0))^{\beta}}{|\zeta| \Gamma(\alpha+\beta)}\right) \int_{0}^{1}|u-\tilde{u}| \mathrm{d} A_{2}(s) \\
& \leq\left[\rho \mathscr{G}_{2}+a_{1}\left(1+\frac{(\psi(1)-\psi(0))^{\beta}}{|\zeta| \Gamma(\alpha+\beta)}\right) \int_{0}^{1} \mathrm{~d} A_{1}(s)\right. \\
& \left.-a_{2}\left(\frac{(\psi(1)-\psi(0))^{\beta}}{|\zeta| \Gamma(\alpha+\beta)}\right) \int_{0}^{1} \mathrm{~d} A_{2}(s)\right]\|u-\widetilde{u}\| \\
& \leq\left(\rho \mathscr{G}_{2}+\mathscr{G}_{3}\right)\|u-\widetilde{u}\| \text {. }
\end{aligned}
$$


By assumption $\rho \mathscr{G}_{2}+\mathscr{G}_{3}<1$, we obtain that $\mathscr{T}_{2}$ is a contraction mapping. Since $f$ and $h$ are continuous functions, we have $\mathscr{T}_{1}$ as continuous. Also, $\mathscr{T}_{1}$ is uniformly bounded on $B_{\epsilon}$ as

$$
\begin{aligned}
\left\|\mathscr{T}_{1} u\right\|= & \sup _{t \in[0,1]}\left|I_{0^{+}, \psi}^{\alpha+\beta} f(t, u(t))-I_{0^{+}, \psi}^{\beta} h(t, u(t))\right| \\
\leq & \sup _{t \in[0,1]}\left\{\int_{0}^{t} \frac{(\psi(t)-\psi(s))^{\alpha+\beta-1}}{\Gamma(\alpha+\beta)} \psi(s)|f(s, u(s))| \mathrm{d} s\right. \\
& \left.-\int_{0}^{t} \frac{(\psi(t)-\psi(s))^{\beta-1}}{\Gamma(\beta)} \psi(s)|h(s, u(s))| \mathrm{d} s\right\} \\
\leq & \|M\|\left(\frac{(\psi(1)-\psi(0))^{\alpha+\beta}}{\Gamma(\alpha+\beta+1)}-\frac{(\psi(1)-\psi(0))^{\beta}}{\Gamma(\beta+1)}\right) .
\end{aligned}
$$

Finally, we prove the compactness of the operator $\mathscr{T}_{1}$. To show this, we define $\sup _{(t, u) \in[0,1] \times B_{\epsilon}}|f(t, u)|=\bar{f}<\infty$ and $\sup _{(t, u) \in[0,1] \times B_{\epsilon}}|h(t, u)|=h<\infty$ and take $0 \leq t_{1}<t_{2} \leq 1$. Thus, we have

$$
\begin{aligned}
& \left|\mathscr{T}_{1} u\left(t_{2}\right)-\mathscr{T}_{1} u\left(t_{1}\right)\right| \leq \int_{0}^{t_{1}} \frac{\left[\left(\psi\left(t_{2}\right)-\psi(s)\right)^{\alpha+\beta-1}-\left(\psi\left(t_{1}\right)-\psi(s)\right)^{\alpha+\beta-1}\right]}{\Gamma(\alpha+\beta)} \\
& \psi(s)|f(s, u(s))| \mathrm{d} s \\
& +\int_{t_{1}}^{t_{2}} \frac{\left(\psi\left(t_{2}\right)-\psi(s)\right)^{\alpha+\beta-1}}{\Gamma(\alpha+\beta)} \psi(s)|f(s, u(s))| \mathrm{d} s \\
& -\int_{0}^{t_{1}} \frac{\left[\left(\psi\left(t_{2}\right)-\psi(s)\right)^{\beta-1}-\left(\psi\left(t_{1}\right)-\psi(s)\right)^{\beta-1}\right]}{\Gamma(\beta)} \\
& \cdot \dot{\psi}(s)|h(s, u(s))| \mathrm{d} s \\
& -\int_{t_{1}}^{t_{2}} \frac{\left(\psi\left(t_{2}\right)-\psi(s)\right)^{\beta-1}}{\Gamma(\beta)} \psi(s)|h(s, u(s))| \mathrm{d} s \\
& \leq \frac{\bar{f}}{\Gamma(\alpha+\beta+1)}\left[2\left(\left(\psi\left(t_{2}\right)-\psi\left(t_{1}\right)\right)^{\alpha+\beta}\right)\right. \\
& \left.+\left(\psi\left(t_{2}\right)-\psi(0)\right)^{\alpha+\beta}-\left(\psi\left(t_{1}\right)-\psi(0)\right)^{\alpha+\beta}\right] \\
& +\frac{\bar{h}}{\Gamma(\beta+1)}\left[2\left(\left(\psi\left(t_{2}\right)-\psi\left(t_{1}\right)\right)^{\beta}\right)-\left(\psi\left(t_{2}\right)-\psi(0)\right)^{\beta}\right. \\
& \left.+\left(\psi\left(t_{1}\right)-\psi(0)\right)^{\beta}\right] \longrightarrow 0, \quad \text { as } t_{2}-t_{1} \longrightarrow 0,
\end{aligned}
$$

independent of $u \in B_{\epsilon}$. Thus, $\mathscr{T}_{1}$ is equicontinuous. So, $\mathscr{T}_{1}$ is relatively compact on $B_{\epsilon}$. Hence, by the Arzela-Ascoli theorem, $\mathscr{T}_{1}$ is compact on $B_{\epsilon}$. Thus, the hypotheses of Theorem 6 are satisfied which leads problem (1) to have at least one solution on $[0,1]$.

Now, we apply Leray-Schauder nonlinear alternative fixed-point theorem to establish an existence result for problem (1).
Theorem 4. Let $f, h:[0,1] \times \mathbb{R} \longrightarrow \mathbb{R}$ be continuous functions and the following conditions are satisfied:

(H3) There exist functions $l_{1}, l_{2} \in C([0,1], \mathbb{R})$ with $l=$ $\max \left\{l_{1}, l_{2}\right\}$ and nondecreasing functions $q_{1}, q_{2}$ : $\mathbb{R}^{+} \longrightarrow \mathbb{R}^{+}, q=\max \left\{q_{1}, q_{2}\right\}$ such that

$$
\begin{aligned}
& |f(t, u)| \leq l_{1} q_{1}(\|u\|), \\
& |h(t, u)| \leq l_{2} q_{2}(\|u\|), \quad \text { for all }(t, u) \in[0,1] \times \mathbb{R} .
\end{aligned}
$$


(H4) There exists a constant $N>0$ such that

$$
\frac{N}{\|l\| q(N) \mathscr{G}+\mathscr{G}_{1}}>1 .
$$

Proof. Let us show that the operator $\mathscr{T}$ maps bounded sets into bounded sets in $C([0,1], \mathbb{R})$. For a positive $r$, let $B_{r}=$ $\{u \in C([0,1], \mathbb{R}):\|u\| \leq r\}$ be a bounded set in $C([0,1], \mathbb{R})$. For each $t \in[0,1]$, by $(\mathrm{H} 3)$, we have

Then, problem (1) has at least one solution on $[0,1]$.

$$
\begin{aligned}
& |(\mathscr{T} u)(t)| \leq \sup _{t \in[0,1]}\left\{\int_{0}^{t} \frac{(\psi(t)-\psi(s))^{\alpha+\beta-1}}{\Gamma(\alpha+\beta)} \psi(s)|f(s, u(s))| \mathrm{d} s\right. \\
& -\int_{0}^{t} \frac{(\psi(t)-\psi(s))^{\beta-1}}{\Gamma(\beta)} \psi(s)|h(s, u(s))| \mathrm{d} s \\
& +\frac{(\psi(t)-\psi(0))^{\beta}}{|\zeta| \Gamma(\alpha+\beta)}\left[\int_{0}^{1} \frac{(\psi(1)-\psi(s))^{\alpha+\beta-1}}{\Gamma(\alpha+\beta)} \psi(s)|f(s, u(s))| \mathrm{d} s\right. \\
& -\int_{0}^{1} \frac{(\psi(1)-\psi(s))^{\beta-1}}{\Gamma(\beta)} \psi(s)|h(s, u(s))| \mathrm{d} s+\left|a_{3}\right| \sum_{i=1}^{m}\left|\mu_{i}\right| \\
& \cdot\left(\int_{0}^{\eta_{i}} \frac{\left(\psi\left(\eta_{i}\right)-\psi(s)\right)^{\alpha+\beta+\gamma-1}}{\Gamma(\alpha+\beta+\gamma)} \psi(s)|f(s, u(s))| \mathrm{d} s\right. \\
& \left.-\int_{0}^{\eta_{i}} \frac{\left(\psi\left(\eta_{i}\right)-\psi(s)\right)^{\beta+\gamma-1}}{\Gamma(\beta+\gamma)} \psi(s)|h(s, u(s))||d| s\right) \\
& \left.\left.+\left|a_{1} \varphi \Omega[u]\right|-\left|a_{2} \theta[u]\right|\right]+\left|a_{1} \Omega[u]\right|\right\} \\
& \leq\left\|l_{1}\right\| q_{1}(\|u\|) \sup _{t \in[0,1]}\left\{\frac{(\psi(t)-\psi(0))^{\alpha+\beta}}{\Gamma(\alpha+\beta+1)}+\frac{(\psi(t)-\psi(0))^{\beta}}{|\zeta| \Gamma(\alpha+\beta)}\right. \\
& \left.\cdot\left(\frac{(\psi(1)-\psi(0))^{\alpha+\beta}}{\Gamma(\alpha+\beta+1)}+\left|a_{3}\right| \sum_{i=1}^{m}\left|\mu_{i}\right| \frac{\left(\psi\left(\eta_{i}\right)-\psi(0)\right)^{\alpha+\beta+\gamma}}{\Gamma(\alpha+\beta+\gamma+1)}\right)\right\} \\
& +\left\|l_{2}\right\| q_{2}(\|u\|) \sup _{t \in[0,1]}\left\{\frac{(\psi(t)-\psi(0))^{\beta}}{|\zeta| \Gamma(\beta+1)}-\frac{(\psi(t)-\psi(0))^{\beta}}{|\zeta| \Gamma(\alpha+\beta)}\right. \\
& \left.\left(\frac{(\psi(1)-\psi(0))^{\beta}}{\Gamma(\beta+1)}+\left|a_{3}\right| \sum_{i=1}^{m}\left|\mu_{i}\right| \frac{\left(\psi\left(\eta_{i}\right)-\psi(0)\right)^{\beta+\gamma}}{\Gamma(\beta+\gamma+1)}\right)\right\} \\
& +a_{1} \Omega[u]\left(1+\frac{(\psi(t)-\psi(0))^{\beta}}{|\zeta| \Gamma(\alpha+\beta)}\right) \\
& \leq\|l\| q(\|r\|)\left\{\frac{(\psi(1)-\psi(0))^{\alpha+\beta}}{\Gamma(\alpha+\beta+1)}-\frac{(\psi(1)-\psi(0))^{\beta}}{\Gamma(\beta+1)}+\frac{(\psi(1)-\psi(0))^{\beta}}{|\zeta| \Gamma(\alpha+\beta)}\right. \\
& \cdot\left[\frac{(\psi(1)-\psi(0))^{\alpha+\beta}}{\Gamma(\alpha+\beta+1)}-\frac{(\psi(1)-\psi(0))^{\beta}}{\Gamma(\beta+1)}+\left|a_{3}\right| \sum_{i=1}^{m}\left|\mu_{i}\right|\right. \\
& \left.\left.\left(\frac{\left(\psi\left(\eta_{i}\right)-\psi(0)\right)^{\alpha+\beta+\gamma}}{\Gamma(\alpha+\beta+\gamma+1)}-\frac{\left(\psi\left(\eta_{i}\right)-\psi(0)\right)^{\beta+\gamma}}{\Gamma(\beta+\gamma+1)}\right)\right]\right\} \\
& +a_{1} \Omega[u]\left(1+\frac{(\psi(1)-\psi(0))^{\beta}}{|\zeta| \Gamma(\alpha+\beta)}\right) \\
& \leq\|l\| q(\|r\|) \mathscr{G}+\mathscr{G}_{1} \text {. }
\end{aligned}
$$


Next, we show that $\mathscr{T}$ maps bounded sets into equicontinuous sets of $C([0,1], \mathbb{R})$. Let $u \in B_{r}$; then, for $t_{1}, t_{2} \in[0,1]$, with $t_{1}<t_{2}$, we have

$$
\begin{aligned}
& \left|\mathscr{T} u\left(t_{2}\right)-\mathscr{T} u\left(t_{1}\right)\right| \leq \int_{0}^{t_{1}} \frac{\left[\left(\psi\left(t_{2}\right)-\psi(s)\right)^{\alpha+\beta-1}-\left(\psi\left(t_{1}\right)-\psi(s)\right)^{\alpha+\beta-1}\right]}{\Gamma(\alpha+\beta)} \\
& \text { - } \psi(s)|f(s, u(s))| \mathrm{d} s \\
& +\int_{t_{1}}^{t_{2}} \frac{\left(\psi\left(t_{2}\right)-\psi(s)\right)^{\alpha+\beta-1}}{\Gamma(\alpha+\beta)} \psi(s)|f(s, u(s))| \mathrm{d} s \\
& -\int_{0}^{t_{1}} \frac{\left[\left(\psi\left(t_{2}\right)-\psi(s)\right)^{\beta-1}-\left(\psi\left(t_{1}\right)-\psi(s)\right)^{\beta-1}\right]}{\Gamma(\beta)} \\
& \cdot \psi(s)|h(s, u(s))| \mathrm{d} s \\
& -\int_{t_{1}}^{t_{2}} \frac{\left(\psi\left(t_{2}\right)-\psi(s)\right)^{\beta-1}}{\Gamma(\beta)} \psi(s)|h(s, u(s))| \mathrm{d} s \\
& +\frac{\left[\left(\psi\left(t_{2}\right)-\psi(0)\right)^{\beta}-\left(\psi\left(t_{1}\right)-\psi(0)\right)^{\beta}\right]}{|\zeta| \Gamma(\alpha+\beta)} \\
& {\left[\int_{0}^{1} \frac{(\psi(1)-\psi(s))^{\alpha+\beta-1}}{\Gamma(\alpha+\beta)} \psi(s)|f(s, u(s))| \mathrm{d} s\right.} \\
& -\int_{0}^{1} \frac{(\psi(1)-\psi(s))^{\beta-1}}{\Gamma(\beta)} \psi(s)|h(s, u(s))| \mathrm{d} s \\
& +\left|a_{3}\right| \sum_{i=1}^{m}\left|\mu_{i}\right|\left(\int_{0}^{\eta_{i}} \frac{\left(\psi\left(\eta_{i}\right)-\psi(s)\right)^{\alpha+\beta+\gamma-1}}{\Gamma(\alpha+\beta+\gamma)} \psi(s)|f(s, u(s))| \mathrm{d} s\right. \\
& \left.-\int_{0}^{\eta_{i}} \frac{\left(\psi\left(\eta_{i}\right)-\psi(s)\right)^{\beta+\gamma-1}}{\Gamma(\beta+\gamma)} \psi(s)|h(s, u(s))| \mathrm{d} s\right) \\
& \left.+\left|a_{1} \varphi \Omega[u]\right|-\left|a_{2} \theta[u]\right|\right] \\
& \leq\left\|l_{1}\right\| q_{1}(\|r\|)\left[\frac{2\left(\psi\left(t_{2}\right)-\psi\left(t_{1}\right)\right)^{\alpha+\beta}+\left(\psi\left(t_{2}\right)-\psi(0)\right)^{\alpha+\beta}-\left(\psi\left(t_{1}\right)-\psi(0)\right)^{\alpha+\beta}}{\Gamma(\alpha+\beta+1)}\right. \\
& +\frac{\left[\left(\psi\left(t_{2}\right)-\psi(0)\right)^{\beta}-\left(\psi\left(t_{1}\right)-\psi(0)\right)^{\beta}\right]}{|\zeta| \Gamma(\alpha+\beta)}\left(\frac{(\psi(1)-\psi(0))^{\alpha+\beta}}{\Gamma(\alpha+\beta+1)}\right. \\
& \left.\left.+\left|a_{3}\right| \sum_{i=1}^{m}\left|\mu_{i}\right| \frac{\left(\psi\left(\eta_{i}\right)-\psi(0)\right)^{\alpha+\beta+\gamma}}{\Gamma(\alpha+\beta+\gamma+1)}\right)\right]+\left\|l_{2}\right\| q_{2}(\|r\|) \\
& \cdot\left[\frac{2\left(\psi\left(t_{2}\right)-\psi\left(t_{1}\right)\right)^{\beta}-\left(\psi\left(t_{2}\right)-\psi(0)\right)^{\beta}+\left(\psi\left(t_{1}\right)-\psi(0)\right)^{\beta}}{\Gamma(\beta+1)}\right. \\
& \left.-\frac{\left[\left(\psi\left(t_{2}\right)-\psi(0)\right)^{\beta}-\left(\psi\left(t_{1}\right)-\psi(0)\right)^{\beta}\right]}{|\zeta| \Gamma(\alpha+\beta)}\left(\frac{(\psi(1)-\psi(0))^{\beta}}{\Gamma(\beta+1)}+\left|a_{3}\right| \sum_{i=1}^{m}\left|\mu_{i}\right| \frac{\left(\psi\left(\eta_{i}\right)-\psi(0)\right)^{\beta+\gamma}}{\Gamma(\beta+\gamma+1)}\right)\right] \text {, }
\end{aligned}
$$


which tends to zero independent of $u \in B_{r}$ as $t_{2}-t_{1} \longrightarrow 0$. So, we deduce that the operator $\mathscr{T}: C([0,1], \mathbb{R}) \longrightarrow C([0,1], \mathbb{R})$ is completely continuous (by Arzela-Ascoli theorem). It remains to show the boundedness of the set of all solutions $u=\lambda \mathscr{T}(u)$ for $\lambda \in(0,1)$. Let $u \in U$ be a solution of problem (1). So, for $t \in[0,1]$, we obtain

$$
|u(t)|=|\lambda(\mathscr{T} u)(t)| \leq|(\mathscr{T} u)(t)|,
$$

which, on taking the norm for $t \in[0,1]$, yields

$$
\|u\| \leq\|l\| q(\|r\|) \mathscr{G}+\mathscr{G}_{1},
$$

and then

$$
\frac{\|u\|}{\|l\| q(\|r\|) \mathscr{G}+\mathscr{G}_{1}} \leq 1
$$

From (H4), we can find $N>0$ such that $\|u\| \neq N$. Take a set $\mathscr{U}_{*}=\{u \in C([0,1], \mathbb{R}):\|u\|<N\}$ and notice that the operator $\mathscr{T}: \overline{\mathscr{U}} \longrightarrow C([0,1], \mathbb{R})$ is continuous and completely continuous. By choice of $\mathcal{U}$, we cannot find a $u \in \partial \mathscr{U}$ such that $u=\lambda \mathscr{T}(u)$ for some $\lambda \in(0,1)$. Hence, by Theorem 2 , the operator $\mathscr{T}$ has a fixed point $u \in \bar{U}$ which is a solution of problem (1).

Example 1. Consider the following boundary value problem:

$$
\left\{\begin{array}{l}
{ }^{c} D_{0^{+}, \psi}^{(1 / 4)}\left({ }^{c} D_{0^{+}, \psi}^{(1 / 2)} u(t)+h(t, u(t))\right)=f(t, u(t)), \quad 0<\alpha, \beta \leq 1, \\
u(0)=a_{1} \Omega[u] \\
u(1)=a_{2} \theta[u]+a_{3} \sum_{i=1}^{2} \mu_{i} I_{0^{+}, \psi}^{\gamma} u\left(\eta_{i}\right),
\end{array}\right.
$$

where

$$
\alpha=(1 / 4), \beta=(1 / 2), A_{1}(s)=A_{2}
$$

$(s)=s, a_{1}=a_{2}=(3 / 4), a_{3}=1, m=2$,

$\mu_{1}=(1 / 5), \mu_{2}=(2 / 5), \eta_{1}=(1 / 6), \eta_{2}=(5 / 6), \gamma=(1 / 3)$, and $\psi(t)=2 t^{2}+1$. Clearly, $\psi$ is an increasing function on $[0,1]$ and $\psi(t)=4 t$ is a continuous function on $[0,1]$.

To illustrate the application of Theorem 3, we take

$$
\begin{aligned}
& f(t, u)=\frac{\cos u}{22 \sqrt{74}}+\frac{e^{-t}}{4 t^{2}+20}, \\
& h(t, u)=\frac{1}{48}\left(\sin u+\frac{5 t^{2}}{2}\right),
\end{aligned}
$$

where $f$ and $h$ satisfy the assumption of Theorem 3. By using the given data, we find $\rho=0.0208, \zeta=0.589473482$,
$M=0.072916, \mathscr{G}_{2}=0.3951268038$, and $\mathscr{G}_{3}=0$. In addition, $\rho \mathscr{G}_{2}+\mathscr{G}_{3} \approx 0.008218637519<1$. Therefore, the result of Theorem 3 applies to problem (38) with $f(t, u)$ and $h(t, u)$ given above.

\section{Existence Results for Inclusion Case}

In this section, we extend the results to cover the inclusion problem and prove the existence of solutions for problem (2) by applying the fixed-point theorem [23]. We recall some basic notations for the inclusion case [24-30].

For a normed space $(X,\|\cdot\|)$, let

$$
\begin{array}{r}
\mathscr{P}_{\mathrm{cl}}(X)=\{y \in \mathscr{P}(x): y \text { is closed }\}, \\
\mathscr{P}_{\text {cp,cv }}(\mathbb{R})=\{\gamma \in \mathscr{P}(\mathbb{R}): \gamma \text { is compact and convex }\} .
\end{array}
$$

A multivalued map $F:[0,1] \times \mathbb{R} \longrightarrow \mathscr{P}(\mathbb{R})$ is said to be caratheodory if

(i) $t \longrightarrow F(t, x)$ is measurable for each $x \in \mathbb{R}$

(ii) $x \longrightarrow F(t, x)$ is upper semicontinuous for almost all $t \in[0,1]$

Furthermore, a caratheodory function $F$ is called $L^{1}$-Caratheodory if

(iii) for each $\alpha>0$, there exist $\varphi_{\alpha} \in L^{1}\left([0,1], \mathbb{R}^{+}\right)$such that

$$
\begin{aligned}
& \qquad\|F(t, x)\|=\sup \{|v|: v \in F(t, x)\} \leq \varphi_{\alpha}(t), \\
& \text { for all }\|x\|_{\infty} \leq \alpha \text { and for a.e. } t \in[0,1] \text {. }
\end{aligned}
$$
by

For each $y \in C([0,1], \mathbb{R})$, define the set of selections of $F$

$$
S_{F, y}:=\left\{v \in L^{1}\left([0,1], \mathbb{R}^{+}\right): v(t) \in F(t, y(t)) \text {, for a.e. } t \in[0,1]\right\} .
$$

Let $(X, d)$ be a metric space induced from the normed space $(X ;\|\cdot\|)$. We have $H_{d}: \mathscr{P}(X) \times \mathscr{P}(X) \longrightarrow \mathbb{R} \cup\{\infty\}$ given by

$$
H_{d}(A, B)=\max \left\{\sup _{a \in A} d(a, B), \sup _{b \in B} d(A, b)\right\},
$$

where $d(A, b)=\inf _{a \in A} d(a ; b)$ and $d(a, B)=\inf _{b \in B} d(a ; b)$. So, $\left(\mathscr{P}_{\mathrm{cl}}(X), H_{d}\right)$ is a metric space [31].

Lemma 4. Let $X$ be a Banach, space. Let $F:[0, T] \times \mathbb{R} \longrightarrow \mathscr{P}_{c p, c v}(X)$ be an $L^{1}$-Caratheodory multivalued map, and let $\Theta$ be a linear continuous mapping from $L^{1}([0,1], X)$ to $C([0,1], X)$. Then, the operator

$$
\Theta^{\circ} S_{F}: C([0,1], X) \longrightarrow \mathscr{P}_{\text {cp }, \mathrm{cv}}(C([0,1], X)), \quad x \longrightarrow\left(\Theta^{\circ} S_{F}\right)(x)=\Theta\left(S_{F, x}\right)
$$

is a closed graph operator in $C([0,1], X) \times C([0,1], X)$.

Lemma 5. If $F: X \longrightarrow \mathscr{P}_{c l}(Y)$ is u.s.c., then $F_{r}(F)$ is a closed subset of $X \times Y$; i.e., for every sequence $\left\{x_{n}\right\}_{n \in \mathbb{N}} \subset X$ and $\left\{y_{n}\right\}_{n \in \mathbb{N}} \subset Y$, if $n \longrightarrow \infty, \quad x_{n} \longrightarrow x_{*}, y_{n} \longrightarrow y_{*}$, and $y_{n} \in F\left(x_{n}\right)$, then $y_{*} \in F\left(x_{*}\right)$. Conversely, if $F$ is completely continuous and has a closed graph, then it is upper semicontinuous. 
Definition 5. A function $u \in C([0,1], \mathbb{R})$ is called a solution of problem (2) if we can find a function $f \in L^{1}([0,1], \mathbb{R})$ with $f(t) \in F(t, u)$ a.e. on $[0,1]$ such that $u(0)=a_{1} \Omega[u]$, $u(1)=a_{2} \theta[u]+a_{3} \sum_{i=1}^{m} \mu_{i} I_{0^{+}, \psi}^{\gamma} u\left(\eta_{i}\right)$ and

$$
\begin{aligned}
u(t)= & I_{0^{+}, \psi}^{\alpha+\beta} f(t)-I_{0^{+}, \psi}^{\beta} h(t)+\frac{(\psi(t)-\psi(0))^{\beta}}{\zeta \Gamma(\beta+1)}\left[I_{0^{+}, \psi}^{\alpha+\beta} f(1)-I_{0^{+}, \psi}^{\beta} h(1)+a_{3} \sum_{i=1}^{m} \mu_{i}\left(I_{0^{+}, \psi}^{\alpha+\beta+\gamma} f\left(\eta_{i}\right)-I_{0^{+}, \psi}^{\beta+\gamma} h\left(\eta_{i}\right)\right)\right. \\
& \left.+a_{1} \varphi \Omega[u]-a_{2} \theta[u]\right]+a_{1} \Omega[u] .
\end{aligned}
$$

For convenience, we denote

$$
\begin{gathered}
\Delta_{1}=\frac{(\psi(1)-\psi(0))^{\beta}}{\Gamma(\beta+1)}+\frac{(\psi(1)-\psi(0))^{\beta}}{|\zeta| \Gamma(\alpha+\beta)}\left(\frac{(\psi(1)-\psi(0))^{\beta}}{\Gamma(\beta+1)}+\left|a_{3}\right| \sum_{i=1}^{m}\left|\mu_{i}\right| \frac{\left(\psi\left(\eta_{i}\right)-\psi(0)\right)^{\beta+\gamma}}{\Gamma(\beta+\gamma+1)}\right), \\
\Delta_{2}=\frac{(\psi(1)-\psi(0))^{\alpha+\beta}}{\Gamma(\alpha+\beta+1)}+\frac{(\psi(t)-\psi(0))^{\beta}}{|\zeta| \Gamma(\alpha+\beta)}\left(\frac{(\psi(1)-\psi(0))^{\alpha+\beta}}{\Gamma(\alpha+\beta+1)}+\left|a_{3}\right| \sum_{i=1}^{m}\left|\mu_{i}\right| \frac{\left(\psi\left(\eta_{i}\right)-\psi(0)\right)^{\alpha+\beta+\gamma}}{\Gamma(\alpha+\beta+\gamma+1)}\right) .
\end{gathered}
$$

Our result is based on the following fixed-point theorem.

Theorem 5. Let $U$ and $\bar{U}$ be, respectively, the open and closed subsets of Banach space $X$, such that $0 \in U$; let $\chi_{1}(u): \bar{U} \longrightarrow \mathscr{P}_{c p, c v}(X)$ be multivalued and $\chi_{2}(u): \bar{U} \longrightarrow X$ be single-valued such that $\chi_{1}(\bar{U})+\chi_{2}(\bar{U})$ is bounded. Suppose that

(a) $\chi_{2}$ is a contraction with a contraction $k<(1 / 2)$

(b) $\chi_{1}$ is u.s.c and compact

Then, either

(i) the operator inclusion $\lambda x \in \chi_{1} x+\chi_{2} x$ has a solution for $\lambda=1$ or

(ii) there is an element $u \in \partial U$ such that $\lambda u \in \chi_{1} u+\chi_{2} u$ for some $\lambda>1$, where $\partial U$ is the boundary of $U$

Theorem 6. Assume that

(N1) $F:[0,1] \times \mathbb{R} \longrightarrow \mathscr{P}_{c p, c}(\mathbb{R})$ is $L^{1}-$ Caratheodory.

(N2) There exists a continuous function $\omega \in C\left([0,1], \mathbb{R}^{+}\right)$and $\Lambda \in C\left([0,1], \mathbb{R}^{+}\right)$such that
$\|F(t, u)\|_{\mathscr{P}}=\sup \{|u|: u \in F(t, u)\} \leq \Lambda(t) \omega(\|u\|)$

$$
\text { for all }(t, u) \in[0,1] \times \mathbb{R} \text {. }
$$

(N3) Let $h:[0,1] \times \mathbb{R} \longrightarrow \mathbb{R}$ be a continuous functions satisfying

$$
|h(s, u)-h(s, \widetilde{u})| \leq \mathscr{E}|u-\tilde{u}|, \quad \forall u, \tilde{u} \in \mathbb{R} \text { and } \mathscr{E}>0 .
$$

(N4) There exists a number $\tau>0$ such that

$$
\frac{\tau}{\|\Lambda\| \omega(\tau) \mathscr{G}+\mathscr{G}_{1}}<1
$$

where $\mathscr{G}$ and $\mathscr{G}_{1}$ are defined in (21) and (22), respectively. Then, problem (2) has at least one solution on $[0,1]$ if $\mathscr{E} \Delta_{1}<(1 / 2)$.
Proof. Let $D=\{u \in U:\|u\|<\varepsilon\}$ be an open set in U. Define the multivalued operator $\chi_{1}: \bar{D} \longrightarrow \mathscr{P}(U)$ by

$$
\chi_{1}(u)=\left\{z \in U: z(t)=I_{0^{+}, \psi}^{\alpha+\beta} f(t)+\frac{(\psi(t)-\psi(0))^{\beta}}{\zeta \Gamma(\beta+1)}\left(I_{0^{+}, \psi}^{\alpha+\beta} f(1)+a_{3} \sum_{i=1}^{m} \mu_{i} I_{0^{+}, \psi}^{\alpha+\beta+\gamma} f\left(\eta_{i}\right)+a_{1} \varphi \Omega[u]-a_{2} \theta[u]\right)+a_{1} \Omega[u]\right\}
$$


and define the single-valued operator $\chi_{2}: \bar{D} \longrightarrow U$ by

$$
\chi_{2}(u)=\left\{z \in U: z(t)=-I_{0^{+}, \psi}^{\beta} h(t)-\frac{(\psi(t)-\psi(0))^{\beta}}{\zeta \Gamma(\beta+1)}\left(I_{0^{+}, \psi}^{\beta} h(1)+a_{3} \sum_{i=1}^{m} \mu_{i} I_{0^{+}, \psi}^{\beta+\gamma} h\left(\eta_{i}\right)\right)\right\} .
$$

Observe that $\chi=\chi_{1}+\chi_{2}$, and it is given by

$$
\begin{aligned}
\chi(u)= & \left\{z(t) \in C([0,1], \mathbb{R}): z(t)=\int_{0}^{t} \frac{(\psi(t)-\psi(s))^{\alpha+\beta-1}}{\Gamma(\alpha+\beta)} \psi(s) f(s) \mathrm{d} s\right. \\
& -\int_{0}^{t} \frac{(\psi(t)-\psi(s))^{\beta-1}}{\Gamma(\beta)} \psi(s) h(s) \mathrm{d} s \\
& +\frac{(\psi(t)-\psi(0))^{\beta}}{\zeta \Gamma(\alpha+\beta)}\left[\int_{0}^{1} \frac{(\psi(1)-\psi(s))^{\alpha+\beta-1}}{\Gamma(\alpha+\beta)} \psi(s) f(s) \mathrm{d} s\right. \\
& -\int_{0}^{1} \frac{(\psi(1)-\psi(s))^{\beta-1}}{\Gamma(\beta)} \psi(s) h(s) \mathrm{d} s \\
& +a_{3} \sum_{i=1}^{m} \mu_{i}\left(\int_{0}^{\eta_{i}} \frac{\left(\psi\left(\eta_{i}\right)-\psi(s)\right)^{\alpha+\beta+\gamma-1}}{\Gamma(\alpha+\beta+\gamma)} \psi(s) f(s) \mathrm{d} s\right. \\
& \left.-\int_{0}^{\eta_{i}} \frac{\left(\psi\left(\eta_{i}\right)-\psi(s)\right)^{\beta+\gamma-1}}{\Gamma(\beta+\gamma)} \psi(s) h(s) \mathrm{d} s\right) \\
& \left.\left.+a_{1} \varphi \Omega[u]-a_{2} \theta[u]\right]+a_{1} \Omega[u], f \in S_{F, u}\right\} .
\end{aligned}
$$

Indeed, if $z \in \chi(u)$, then there exists $f \in S_{F, u}$, such that

$$
S_{F, u}=\left\{f \in L^{1}\left([0,1], \mathbb{R}^{+}\right): f(t) \in F(t, u(t)) \text {, for a.e. } t \in[0,1]\right\} .
$$

We will show that the maps $\chi_{1}$ and $\chi_{2}$ satisfy the hypotheses of Theorem 5. This will be done in several steps. 
Step 1. $\chi_{2}$ is a contraction. Let $u, \tilde{u} \in \mathbb{R}$, by (N3), we have

$$
\begin{aligned}
\left\|\chi_{2} u-\chi_{2} \tilde{u}\right\| \leqslant & \sup _{t \in[0,1]}\left\{-\int_{0}^{t} \frac{(\psi(t)-\psi(s))^{\beta-1}}{\Gamma(\beta)} \psi(s)|h(s, u(s))-h(s, \widetilde{u}(s))| \mathrm{d} s\right. \\
& -\frac{(\psi(t)-\psi(0))^{\beta}}{|\zeta| \Gamma(\alpha+\beta)}\left(\int_{0}^{1} \frac{(\psi(1)-\psi(s))^{\beta-1},}{\Gamma(\beta)} \psi(s)|h(s, u(s))-h(s, \tilde{u}(s))| \mathrm{d} s\right. \\
& \left.\left.+\left|a_{3}\right| \sum_{i=1}^{m}\left|\mu_{i}\right| \int_{0}^{\eta_{i}} \frac{\left(\psi\left(\eta_{i}\right)-\psi(s)\right)^{\beta+\gamma-1}}{\Gamma(\beta+\gamma)} \psi(s)|h(s, u(s))-h(s, \tilde{u}(s))| \mathrm{d} s\right)\right\} \\
\leq & \mathscr{E}\|u-\widetilde{u}\| \sup _{t \in[0,1]}\left\{-\frac{(\psi(t)-\psi(0))^{\beta}}{\Gamma(\beta+1)}-\frac{(\psi(t)-\psi(0))^{\beta}}{|\zeta| \Gamma(\alpha+\beta)}\left(\frac{(\psi(t)-\psi(0))^{\beta}}{\Gamma(\beta+1)}\right.\right. \\
& \left.\left.\left.+\left|a_{3}\right| \sum_{i=1}^{m}\left|\mu_{i}\right| \frac{\left(\psi\left(\eta_{i}\right)-\psi(0)\right)^{\beta+\gamma}}{\Gamma(\beta+\gamma+1)}\right)\right]\right\} \\
\leq & \mathscr{E}\|u-\widetilde{u}\|\left[\frac{(\psi(1)-\psi(0))^{\beta}}{\Gamma(\beta+1)}+\frac{(\psi(1)-\psi(0))^{\beta}}{|\zeta| \Gamma(\alpha+\beta)}\left(\frac{(\psi(1)-\psi(0))^{\beta}}{\Gamma(\beta+1)}\right.\right. \\
& \left.\left.+\left|a_{3}\right| \sum_{i=1}^{m}\left|\mu_{i}\right| \frac{\left(\psi\left(\eta_{i}\right)-\psi(0)\right)^{\beta+\gamma}}{\Gamma(\beta+\gamma+1)}\right)\right] \\
\leq & \mathscr{L} \Delta_{1}\|u-\widetilde{u}\|,
\end{aligned}
$$

which proves that $\chi_{2}$ is a contraction map.

Step 2. $\chi_{1}(u)$ is convex for all $u \in \bar{D}$. Let $z_{1}, z_{2} \in \chi_{1}(u)$. We select $f_{1}, f_{2} \in S_{F, u}$ such that, for each $t \in[0,1]$, we obtain

$$
z_{i}(t)=I_{0^{+}, \psi}^{\alpha+\beta} f_{i}(t)+\frac{(\psi(t)-\psi(0))^{\beta}}{\zeta \Gamma(\beta+1)}\left(I_{0^{+}, \psi}^{\alpha+\beta} f_{i}(1)+a_{3} \sum_{i=1}^{m} \mu_{i} I_{0^{+}, \psi}^{\alpha+\beta+\gamma} f_{i}\left(\eta_{i}\right)+a_{1} \varphi \Omega[u]-a_{2} \theta[u]\right)+a_{1} \Omega[u]
$$

for $i=1,2$.

$$
\begin{aligned}
{\left[\phi z_{1}+(1-\phi) z_{2}\right](t)=} & \int_{0}^{t} \frac{(\psi(t)-\psi(s))^{\alpha+\beta-1}}{\Gamma(\alpha+\beta)} \psi(s)\left[\phi f_{1}(s)+(1-\phi) f_{2}(s)\right] \mathrm{d} s \\
& +\frac{(\psi(t)-\psi(0))^{\beta}}{|\zeta| \Gamma(\alpha+\beta)}\left(\int_{0}^{1} \frac{(\psi(1)-\psi(s))^{\alpha+\beta-1}}{\Gamma(\alpha+\beta)} \psi(s)\right. \\
& \cdot\left[\phi f_{1}(s)+(1-\phi) f_{2}(s)\right] \mathrm{d} s+\left|a_{3}\right| \sum_{i=1}^{m}\left|\mu_{i}\right| \\
& \cdot \int_{0}^{\eta_{i}} \frac{\left(\psi\left(\eta_{i}\right)-\psi(s)\right)^{\alpha+\beta+\gamma-1}}{\Gamma(\alpha+\beta+\gamma)} \psi(s) \\
& \cdot\left[\phi f_{1}(s)+(1-\phi) f_{2}(s)\right] \mathrm{d}+a_{1}[\phi+(1-\phi)] \varphi \Omega[u] \\
& \left.-a_{2}[\phi+(1-\phi)] \theta[u]\right)+a_{1}[\phi+(1-\phi)] \Omega[u] .
\end{aligned}
$$


Since $S_{F, u}$ is convex, it follows that $\phi z_{1}+(1-\phi) z_{2} \in \chi_{1}(u)$ and then $\chi_{1}(u)$ is convex-valued.

Step 3. $\chi_{1}$ is compact and upper semicontinuous. This will be done in various statements. First, we show that $\chi_{1}$ maps bounded sets into bounded sets in $U$.
For a positive number $\varsigma$, let $B_{\varsigma}=\{u \in U:\|u\| \leq \varsigma\}$ be a bounded ball in $\bar{D}$. So, for all $z \in \chi_{1}, u \in B_{\varsigma}$, there exists $f \in S_{F, u}$ such that

$$
z(t)=I_{0^{+}, \psi}^{\alpha+\beta} f(t)+\frac{(\psi(t)-\psi(0))^{\beta}}{\zeta \Gamma(\beta+1)}\left(I_{0^{+}, \psi}^{\alpha+\beta} f(1)+a_{3} \sum_{i=1}^{m} \mu_{i} I_{0^{+}, \psi}^{\alpha+\beta+\gamma} f\left(\eta_{i}\right)+a_{1} \varphi \Omega[u]-a_{2} \theta[u]\right)+a_{1} \Omega[u]
$$

By using (N2), for each $t \in[0,1]$, we have

$$
\begin{aligned}
|z(t)| \leq & \int_{0}^{t} \frac{(\psi(t)-\psi(s))^{\alpha+\beta-1}}{\Gamma(\alpha+\beta)} \psi(s)|f(s, u(s))| \mathrm{d} s \\
& +\frac{(\psi(t)-\psi(0))^{\beta}}{|\zeta| \Gamma(\alpha+\beta)}\left(\int_{0}^{1} \frac{(\psi(1)-\psi(s))^{\alpha+\beta-1},}{\Gamma(\alpha+\beta)} \psi(s)|f(s, u(s))| \mathrm{d} s\right. \\
& +\left|a_{3}\right| \sum_{i=1}^{m}\left|\mu_{i}\right| \int_{0}^{\eta_{i}} \frac{\left(\psi\left(\eta_{i}\right)-\psi(s)\right)^{\alpha+\beta+\gamma-1},}{\Gamma(\alpha+\beta+\gamma)} \psi(s)|f(s, u(s))| \mathrm{d} s \\
& \left.+\left|a_{1} \varphi \Omega[u]\right|-\left|a_{2} \theta[u]\right|\right)+\left|a_{1} \Omega[u]\right| \\
\leq & \|\Lambda\| \omega(\|u\|)\left[\frac{(\psi(1)-\psi(0))^{\alpha+\beta}}{\Gamma(\alpha+\beta+1)}+\frac{(\psi(t)-\psi(0))^{\beta}}{|\zeta| \Gamma(\alpha+\beta)}\left(\frac{(\psi(1)-\psi(0))^{\alpha+\beta}}{\Gamma(\alpha+\beta+1)}\right.\right. \\
& \left.\left.+\left|a_{3}\right| \sum_{i=1}^{m}\left|\mu_{i}\right| \frac{\left(\psi\left(\eta_{i}\right)-\psi(0)\right)^{\alpha+\beta+\gamma}}{\Gamma(\alpha+\beta+\gamma+1)}\right)\right]+a_{1} \Omega[u]\left(1+\frac{(\psi(1)-\psi(0))^{\beta}}{|\zeta| \Gamma(\alpha+\beta)}\right)
\end{aligned}
$$

Consequently,

$$
\|z\| \leq\|\Lambda\| \oplus(\|u\|) \Delta_{2}+\mathscr{G}_{1}
$$

where $\mathscr{G}_{1}$ and $\Delta_{2}$ are defined in (22) and (47), respectively. Second, we prove that $\chi_{1}$ maps bounded sets into equicontinuous sets. Let $\tau_{1}, \tau_{2} \in[0,1]$ with $\tau_{1}<\tau_{2}$ and $u \in B_{\zeta}$; we have 


$$
\begin{aligned}
\left|z\left(\tau_{2}\right)-z\left(\tau_{1}\right)\right| \leq & \int_{0}^{\tau_{1}} \frac{\left[\left(\psi\left(\tau_{2}\right)-\psi(s)\right)^{\alpha+\beta-1}-\left(\psi\left(\tau_{1}\right)-\psi(s)\right)^{\alpha+\beta-1}\right]}{\Gamma(\alpha+\beta)} \psi(s)|f(s, u(s))| \mathrm{d} s \\
& +\int_{\tau_{1}}^{\tau_{2}} \frac{\left(\psi\left(\tau_{2}\right)-\psi(s)\right)^{\alpha+\beta-1}}{\Gamma(\alpha+\beta)} \psi(s)|f(s, u(s))| \mathrm{d} s \\
& +\frac{\left[\left(\psi\left(\tau_{2}\right)-\psi(0)\right)^{\beta}-\left(\psi\left(\tau_{1}\right)-\psi(0)\right)^{\beta}\right]}{|\zeta| \Gamma(\alpha+\beta)} \\
& \cdot\left(\int_{0}^{1} \frac{(\psi(1)-\psi(s))^{\alpha+\beta-1}}{\Gamma(\alpha+\beta)} \psi(s)|f(s, u(s))| \mathrm{d} s+\left|a_{3}\right| \sum_{i=1}^{m}\left|\mu_{i}\right|\right. \\
& \left.\cdot \int_{0}^{\eta_{i}} \frac{\left(\psi\left(\eta_{i}\right)-\psi(s)\right)^{\alpha+\beta+\gamma-1}}{\Gamma(\alpha+\beta+\gamma)} \psi(s)|f(s, u(s))| \mathrm{d} s\right) \\
\leq & \frac{2\left(\psi\left(\tau_{2}\right)-\psi\left(\tau_{1}\right)\right)^{\alpha+\beta}+\left(\psi\left(\tau_{2}\right)-\psi(0)\right)^{\alpha+\beta}-\left(\psi\left(\tau_{1}\right)-\psi(0)\right)^{\alpha+\beta}}{\Gamma(\alpha+\beta+1)} \\
& +\frac{\left[\left(\psi\left(\tau_{2}\right)-\psi(0)\right)^{\beta}-\left(\psi\left(\tau_{1}\right)-\psi(0)\right)^{\beta}\right]}{|\zeta| \Gamma(\alpha+\beta)}\left(\frac{(\psi(1)-\psi(0))^{\alpha+\beta}}{\Gamma(\alpha+\beta+1)}\right. \\
& \left.+\left|a_{3}\right| \sum_{i=1}^{m}\left|\mu_{i}\right| \frac{\left(\psi\left(\eta_{i}\right)-\psi(0)\right)^{\alpha+\beta+\gamma}}{\Gamma(\alpha+\beta+\gamma+1)}\right) .
\end{aligned}
$$

In the above inequality, the right hand side tends to zero independent of $u \in B_{e}$ as $\tau_{2}-\tau_{1} \longrightarrow 0$. Consequently, by the Arzela-Ascoli theorem, we conclude that $\chi_{1}: \bar{D} \longrightarrow P(U)$ is completely continuous and then $\chi_{1}$ is completely continuous. Finally, we show $\chi_{1}$ has a closed graph. Let $u_{n} \longrightarrow u_{*}, z_{n} \in \chi_{1}\left(u_{n}\right)$, and $z_{n} \longrightarrow z_{*}$. Then, we show that $z_{*} \in \chi_{1}\left(u_{*}\right)$. Since $z_{n} \in \chi_{1}\left(u_{n}\right)$, there exists $z_{n} \in S_{F, u_{n}}$ such that for each $t \in[0,1]$, we find that

$$
z_{n}(t)=I_{0^{+}, \psi}^{\alpha+\beta} f_{n}(t)+\frac{(\psi(t)-\psi(0))^{\beta}}{\zeta \Gamma(\beta+1)}\left(I_{0^{+}, \psi}^{\alpha+\beta} f_{n}(1)+a_{3} \sum_{i=1}^{m} \mu_{i} I_{0^{+}, \psi}^{\alpha+\beta+\gamma} f_{n}\left(\eta_{i}\right)+a_{1} \varphi \Omega[u]-a_{2} \theta[u]\right)+a_{1} \Omega[u] .
$$

Now, we have to show that there exists $z * \in S_{F, u_{*}}$ such that for each $t \in[0,1]$,

$$
z_{*}(t)=I_{0^{+}, \psi}^{\alpha+\beta} f_{*}(t)+\frac{(\psi(t)-\psi(0))^{\beta}}{\zeta \Gamma(\beta+1)}\left(I_{0^{+}, \psi}^{\alpha+\beta} f_{*}(1)+a_{3} \sum_{i=1}^{m} \mu_{i} I_{0^{+}, \psi}^{\alpha+\beta+\gamma} f_{*}\left(\eta_{i}\right)+a_{1} \varphi \Omega[u]-a_{2} \theta[u]\right)+a_{1} \Omega[u] .
$$

Consider the continuous linear operator $\Theta: L^{1}([0,1], \mathbb{R}) \longrightarrow U$ given by

$$
f \mapsto \Theta(f)(t)=I_{0^{+}, \psi}^{\alpha+\beta} f(t)+\frac{(\psi(t)-\psi(0))^{\beta}}{\zeta \Gamma(\beta+1)}\left(I_{0^{+}, \psi}^{\alpha+\beta} f(1)+a_{3} \sum_{i=1}^{m} \mu_{i} I_{0^{+}, \psi}^{\alpha+\beta+\gamma} f\left(\eta_{i}\right)+a_{1} \varphi \Omega[u]-a_{2} \theta[u]\right)+a_{1} \Omega[u] .
$$


Note that

$$
\begin{aligned}
\left\|z_{n}(t)-z_{*}(t)\right\|= & \sup _{t \in[0,1]} \mid I_{0^{+}, \psi}^{\alpha+\beta}\left(f_{n}(t)-f_{*}(t)\right)+\frac{(\psi(t)-\psi(0))^{\beta}}{\zeta \Gamma(\beta+1)}\left(I_{0^{+}, \psi}^{\alpha+\beta}\left(f_{n}(1)-f_{*}(1)\right)+a_{3} \sum_{i=1}^{m} \mu_{i} I_{0^{+}, \psi}^{\alpha+\beta+\gamma}\right. \\
& \left.\cdot\left(f_{n}\left(\eta_{i}\right)-f_{*}\left(\eta_{i}\right)\right)+a_{1} \varphi \Omega[u]-a_{2} \theta[u]\right) \mid
\end{aligned}
$$

which goes to 0 , as $n \longrightarrow \infty$.

$$
z_{*}(t)=I_{0^{+}, \psi}^{\alpha+\beta} f_{*}(t)+\frac{(\psi(t)-\psi(0))^{\beta}}{\zeta \Gamma(\beta+1)}\left(I_{0^{+}, \psi}^{\alpha+\beta} f_{*}(1)+a_{3} \sum_{i=1}^{m} \mu_{i} I_{0^{+}, \psi}^{\alpha+\beta+\gamma} f_{*}\left(\eta_{i}\right)+a_{1} \varphi \Omega[u]-a_{2} \theta[u]\right)+a_{1} \Omega[u]
$$

for some $z_{*} \in S_{F, u_{*}}$. Hence, $\chi_{1}$ has a closed graph (and therefore has closed values). Hence, we conclude that $\chi_{1}$ is a compact multivalued map, upper semicontinuous with convex closed values.
It follows by Lemma 5 that $\Theta^{\circ} S_{F}$ is a closed graph operator. Furthermore, we obtain $z_{n}(t) \in \Theta\left(S_{F, u_{n}}\right)$. Since $u_{n} \longrightarrow u_{*}$, we have
Step 4. There exists an open set $Q \subseteq C([0,1], \mathbb{R})$ with $u \notin \lambda \chi_{2}(u)$ for any $\lambda>1$ and for each $u \in \partial Q$. Take $\lambda>1$. Let $u$ be a solution of $(2)$; then, there exists $f \in L^{1}([0,1], \mathbb{R})$ with $f \in S_{f, u}$ such that for $t \in[0,1]$, we have

$$
\begin{aligned}
& u(t)=\frac{(\psi(t)-\psi(s))^{\alpha+\beta-1}}{\Gamma(\alpha+\beta)} \psi(s) f(s) \mathrm{d} s-\int_{0}^{t} \frac{(\psi(t)-\psi(s))^{\beta-1}}{\Gamma(\beta)} \psi(s) h(s) \mathrm{d} s \\
& +\frac{(\psi(t)-\psi(0))^{\beta}}{\zeta \Gamma(\alpha+\beta)}\left[\int_{0}^{1} \frac{(\psi(1)-\psi(s))^{\alpha+\beta-1}}{\Gamma(\alpha+\beta)} \psi(s) f(s) \mathrm{d} s-\int_{0}^{1} \frac{(\psi(1)-\psi(s))^{\beta-1}}{\Gamma(\beta)} \psi(s) h(s) \mathrm{d} s\right. \\
& +a_{3} \sum_{i=1}^{m} \mu_{i}\left(\int_{0}^{\eta_{i}} \frac{\left(\psi\left(\eta_{i}\right)-\psi(s)\right)^{\alpha+\beta+\gamma-1}}{\Gamma(\alpha+\beta+\gamma)} \psi(s) f(s) \mathrm{d} s\right. \\
& \left.\left.-\int_{0}^{\eta_{i}} \frac{\left(\psi\left(\eta_{i}\right)-\psi(s)\right)^{\beta+\gamma-1}}{\Gamma(\beta+\gamma)} \psi(s) h(s) \mathrm{d} s\right)+a_{1} \varphi \Omega[u]-a_{2} \theta[u]\right]+a_{1} \Omega[u] \\
& |u(t)| \leq \int_{0}^{t} \frac{(\psi(t)-\psi(s))^{\alpha+\beta-1}}{\Gamma(\alpha+\beta)} \psi(s)|f(s)| \mathrm{d} s-\int_{0}^{t} \frac{(\psi(t)-\psi(s))^{\beta-1}}{\Gamma(\beta)} \psi(s)|h(s)| \mathrm{d} s \\
& +\frac{(\psi(t)-\psi(0))^{\beta}}{\zeta \Gamma(\alpha+\beta)}\left[\int_{0}^{1} \frac{(\psi(1)-\psi(s))^{\alpha+\beta-1}}{\Gamma(\alpha+\beta)} \psi(s)|f(s)| \mathrm{d} s-\int_{0}^{1} \frac{(\psi(1)-\psi(s))^{\beta-1}}{\Gamma(\beta)} \psi(s)|h(s)| \mathrm{d} s\right. \\
& +a_{3} \sum_{i=1}^{m} \mu_{i}\left(\int_{0}^{\eta_{i}} \frac{\left(\psi\left(\eta_{i}\right)-\psi(s)\right)^{\alpha+\beta+\gamma-1}}{\Gamma(\alpha+\beta+\gamma)} \psi(s)|f(s)| \mathrm{d} s-\int_{0}^{\eta_{i}} \frac{\left(\psi\left(\eta_{i}\right)-\psi(s)\right)^{\beta+\gamma-1}}{\Gamma(\beta+\gamma)} \psi(s)|h(s)| \mathrm{d} s\right) \\
& \left.+a_{1} \varphi \Omega[u]-a_{2} \theta[u]\right]+a_{1} \Omega[u] \\
& \leq\|\Lambda\| \oplus(\|u\|)\left\{\frac{(\psi(1)-\psi(0))^{\alpha+\beta}}{\Gamma(\alpha+\beta+1)}-\frac{(\psi(1)-\psi(0))^{\beta}}{\Gamma(\beta+1)}+\frac{(\psi(1)-\psi(0))^{\beta}}{|\zeta| \Gamma(\alpha+\beta)}\right. \\
& \cdot\left[\frac{(\psi(1)-\psi(0))^{\alpha+\beta}}{\Gamma(\alpha+\beta+1)}-\frac{(\psi(1)-\psi(0))^{\beta}}{\Gamma(\beta+1)}+\left|a_{3}\right| \sum_{i=1}^{m}\left|\mu_{i}\right|\left(\frac{\left(\psi\left(\eta_{i}\right)-\psi(0)\right)^{\alpha+\beta+\gamma}}{\Gamma(\alpha+\beta+\gamma+1)}\right.\right. \\
& \left.\left.-\frac{\left(\psi\left(\eta_{i}\right)-\psi(0)\right)^{\beta+\gamma}}{\Gamma(\beta+\gamma+1)}\right)\right\}+a_{1} \Omega[u]\left(1+\frac{(\psi(1)-\psi(0))^{\beta}}{|\zeta| \Gamma(\alpha+\beta)}\right)
\end{aligned}
$$


which implies

$$
\frac{\|u\|}{\|\Lambda\| \bowtie(\|u\|) \mathscr{G}+\mathscr{G}_{1}}<1
$$

By (N4), there exists $\tau>0$ such that $\|u\| \neq \tau$. Define a set

$$
\mathbb{Q}=\{u \in C([0,1], \mathbb{R}):\|u\|<\tau\} .
$$

Note that the operator $\chi_{1}: \bar{Q} \longrightarrow \mathscr{P}(U)$ is a compact multivalued map, u.s.c. with convex closed values. With the given choice of $Q$, it is not possible to find $u \in \partial Q$ satisfying $u \in \lambda \chi(u)$ for some $\lambda>1$. Consequently, the operator $\chi(u)$ has a fixed point $u \in \bar{Q}$, which is a solution of problem (2).

Example 2. Consider the following boundary value problem:

$$
\left\{\begin{array}{l}
{ }^{c} D_{0^{+}, \psi}^{(1 / 3)}\left({ }^{c} D_{0^{+}, \psi}^{(1 / 4)} u(t)+h(t, u(t))\right) \in F(t, u(t)), \quad 0<\alpha, \beta \leq 1, \\
u(0)=a_{1} \Omega[u], \\
u(1)=a_{2} \theta[u]+a_{3} \sum_{i=1}^{2} \mu_{i} I_{0^{+}, \psi}^{\gamma} u\left(\eta_{i}\right),
\end{array}\right.
$$

where $F: C([0,1], \mathbb{R}) \longrightarrow \mathscr{P}(\mathbb{R})$ is a multivalued map given by

$$
u \longrightarrow F(t, u)=\left[\frac{|u|}{|u|+11}+\frac{(1+2 t)}{9}, \cos u+\frac{(1+t)}{3}\right] .
$$

For $f \in F$, we obtain

$$
|f| \leq \max \left[\frac{|u|}{|u|+11}+\frac{(1+2 t)}{9}, \cos u+\frac{(1+t)}{3}\right] \leq \frac{5}{3} .
$$

Here, $\alpha=(1 / 3), \beta=(1 / 4), A_{1} \quad(s)=A_{2}(s)=s, a_{1}=0$, $a_{2}=(3 / 4), a_{3}=1, m=2$,

$\mu_{1}=(1 / 7), \mu_{2}=(3 / 7), \eta_{1}=(1 / 5), \eta_{2}=(4 / 5), \gamma=(1 / 2)$, and $\psi(t)=3 t^{2}+2$. Clearly, $\psi$ is an increasing function on $[0,1]$ and $\psi(t)=6 t$ is a continuous function on $[0,1]$.

Clearly,

$$
\begin{aligned}
& \|F(t, u)\|_{\mathscr{P}}=\sup \{|u|: u \in F(t, u)\} \leq \Lambda(t) \omega(\|u\|) ; \\
& \text { for all }(t, u) \in[0,1] \times \mathbb{R} \text {, }
\end{aligned}
$$

with $\|\Lambda\|=1$ and $\omega(\|u\|)=(5 / 3)$.

Next, we take

$$
h(t, u)=\frac{4}{217}\left(\sin u+\frac{t^{4}+e^{t}}{26+3 t}\right)
$$

where the function $h$ satisfies the assumption of Theorem 6 . By using the given data, we find $\mathscr{E}=$ $0.0184331797, \zeta=-0.378649448, \Delta_{1}=6.552924874, \quad \mathscr{G}=$ 2.343253024, and $\mathscr{G}_{1}=0$. Thus,

$$
\|u\|>\|\Lambda\| \omega(\|u\|) \mathscr{G}+\mathscr{G}_{1} \approx 3.905421707,
$$

and we have

$$
\mathscr{E} \Delta_{1} \approx 0.1207912419<\frac{1}{2}
$$

Therefore, all the conditions of Theorem 6 are satisfied. Then, there exists at least one solution of problem (70) on $[0,1]$.

\section{Data Availability}

No data were used to support this study.

\section{Conflicts of Interest}

The authors declare no conflicts of interest.

\section{References}

[1] R. Herrmann, Fractional Calculus for Physicist, World Scientific, Singapore, 2014.

[2] A. A. Kilbas, H. M. Srivastava, and J. J. Trujillo, "Thoery and aplications of fractional differential equations," in NorthHoland Mathematics Studies, vol. 24, Elsevier, Amsterdam, Netherlands, 2006.

[3] K. Diethelm, Analysis of Fractional Differential Equations, Springer, Berlin, Germany, Lecture Notes in Mathematics, 2010.

[4] L. Podlubny, Fractional Differential Equations, Academic Press, San Diego, California, 1999.

[5] B. Ross, Ed., in Proceedings Of The International Conference Held at the University Of New Haven, vol. 457, Springer, New Haven, Connecticut, June 1974.

[6] T. M. Atanackovic, S. Pilipovic, B. Stankovic, and D. Zorica, Fractional Calculus with Applications in Mechanics: Wave Propagation,impact and Variational Principles, Wiley, New York, NY, USA, 2014.

[7] O. P. Agrawal, "Some generalized fractional calculus operators and their applications in integral equations," Fractional Calculus and Applied Analysis, vol. 15, pp. 700-711, 2012.

[8] R. Almeida, "A Caputo fractional derivative of a function with respect to another function," Communications in Nonlinear Science and Numerical Simulation, vol. 44, pp. 460-481, 2017.

[9] S. G. Samko, A. A. Kilbas, and O. I. Marichev, Fractional Integrals and Derivatives: Theory and Applications, Gordon and Breach Science Publishers, Switzerland, London, UK, 1993.

[10] R. Almeida, A. B. Malinowska, and M. T. T. Monteiro, "Fractional differential equations with a Caputo derivative with respect to a kernel function and their applications," Mathematical Methods in the Applied Sciences, vol. 41, no. 1, pp. 336-352, 2018.

[11] R. Almeida, "Fractional differential equations with mixed boundary conditions," Bulletin of the Malaysian Mathematical Sciences Society, vol. 42, no. 4, pp. 1687-1697, 2019.

[12] M. S. Abdo, S. K. Panchal, and A. M. Saeed, "Fractional boundary value problem with $\Psi$-Caputo fractional derivative," Proceedings- Mathematical Science, vol. 129, no. 5, p. 65, 2019. 
[13] M. S. Abdo, A. G. Ibrahim, and S. K. Panchal, "Nonlinear implicit fractional differential equation involving $\psi$-Caputo fractional derivative," PJMS, vol. 22, no. 3, 2019.

[14] D. Vivek, E. Elsayed, and K. Kanagarajan, "Theory and analysis of -fractional differential equations with boundary conditions," Communications on Pure and Applied Analysis, vol. 22, pp. 401-414, 2018.

[15] H. A. Wahash, M. S. Abdo, A. M. Saeed, and S. K. Panchal, "Singular fractional differential equations with $\psi$-Caputo operator and modified Picard's iterative method," Applied Mathematics E-Notes, vol. 20, pp. 215-229, 2020.

[16] G. Z. Voyiadjis and W. Sumelka, "Brain modelling in the framework of anisotropic hyperelasticity with time fractional damage evolution governed by the Caputo-Almeida fractional derivative," Journal of the Mechanical Behavior of Biomedical Materials, vol. 89, pp. 209-216, 2019.

[17] J. R. L. Webb and G. Infante, "Positive solutions of nonlocal boundary value problems: a unified approach," Journal of the London Mathematical Society, vol. 74, no. 3, pp. 673-693, 2006.

[18] B. Ahmad, A. Alsaedi, and B. S. Alghamdi, "Analytic approximation of solutions of the forced Duffing equation with integral boundary conditions," Nonlinear Analysis: Real World Applications, vol. 9, no. 4, pp. 1727-1740, 2008.

[19] S. Belmor, C. Ravichandran, and F. Jarad, "Nonlinear generalized fractional differential equations with generalized fractional integral conditions," Journal of Taibah University for Science, vol. 14, no. 1, pp. 114-123, 2020.

[20] B. Ahmad, M. Alghanmi, S. K. Ntouyas, and A. Alsaedi, "Fractional differential equations involving generalized derivative with Stieltjes and fractional integral boundary conditions," Applied Mathematics Letters, vol. 84, pp. 111-117, 2018.

[21] D. R. Smart, Fixed Point Theorems, Cambridge University Press, Cambridge, UK, 1980.

[22] A. Granas and J. Dugundji, Fixed Point Theory, Springer Science and Business Media, New York, NY, USA, 2005.

[23] A. Petrusel, "Fixed points and selections for multivalued operators," Seminar on Fixed Point Theory Cluj-Napoca, vol. 2, pp. 3-22, 2001.

[24] R. P. Agarwal and B. Ahmad, "Existence theory for antiperiodic boundary value problems of fractional differential equations and inclusions," Computers and Mathematics with Applications, vol. 62, no. 3, pp. 1200-1214, 2011.

[25] A. Alsaedi, B. Ahmad, M. Alghanmi, and S. K. Ntouyas, "On a generalized Langevin type nonlocal fractional integral multivalued problem," Mathematics, vol. 7, no. 11, p. 1015, 2019.

[26] C. Castaing and M. Valadier, Convex Analysis and Measurable Multifunctions, p. 580, Springer, New York, NY, USA, 2006.

[27] C. Castaing and M. Valadier, Convex Analysis and Measurable Multifunctions, vol. 580, 1977 Lecture Notes in Mathematics.

[28] K. Deimling, Multivalued Differential Equations, Vol. 1, Walter De Gruyter, Berlin-New York, 1992.

[29] A. Lasota and Z. Opial, "An application of the Kakutani-Ky Fan theorem in the theory of ordinary differential equations," Bulletin L'Académie Polonaise des Science, Série des Sciences Mathématiques, Astronomiques et Physiques, vol. 13, pp. 781-786, 1965.

[30] B. C. Dhage, "Multi-valued mappings and fixed points II," Tamkang Journal of Mathematics, vol. 37, no. 1, pp. 27-46, 2006.

[31] M. Kisielewicz, Differential Inclusions and Optimal Control, Kluwer, Dordrecht, The Netherlands, 1991. 